\title{
Real Time Tracking RGB Color based Kinect
}

\author{
Ahmed Mustafa Taha Alzbier ${ }^{1} \&$ Han cheng ${ }^{1}$ \\ ${ }^{1}$ Changchun University of Science and Technology, Changchun, China \\ Correspondence: Ahmed, Changchun University of Science and Technology, Changchun, China. Tel: \\ 86-1363-0588-381.E-mail: AMT4047@gmail.com \\ Received: September 27, 2016 \\ Accepted: October 6, $2016 \quad$ Online Published: January 14, 2017 \\ doi:10.5539/mas.v11n3p98 \\ URL: http://dx.doi.org/10.5539/mas.v11n3p98
}

\begin{abstract}
As the present computer vision technology is growing up fast, and the multiple RGB color object tracking is considered as one of the important tasks in computer vision and technique that can be used in many applications such as surveillance in a factory production line, event organization, flow control application, analysis and sort by colors and etc. In video processing applications, variants of the background subtraction method are broadly used for the detection of moving objects in video sequences. The background subtraction is the most popular and common approach for motion detection. However, this is paper presents our investigation the first objective of the whole algorithm chain is to find the RGB color within a video. The idea from the beginning was to look for certain specific features of the patches, which would allow distinguishing red, green and blue color objects in the image. In this paper an algorithm is proposed to track the real time moving RGB color objects using kinect camera. We will use a kinect camera to capture the real time video and making an image frame from this video and extracting red, green and blue color .Here image processing is done through MATLAB for color recognition process each color. Our method can tracking accurately at $95 \%$ in real-time.
\end{abstract}

Keywords: Computer Vision, Color Model, Median filter, Blob Analysis, Median filter, Kinect, Segmentation

\section{Introduction}

In computer vision Color object detection and tracking is an important and fundamental topic. Color is a significant of information in image analysis classification. However, Kinect has been developed for Microsoft Kinect version tow console and includes cameras that deliver depth information, color data. An independent developer offers solutions for using Kinect separate from the game console and for the most common operating systems. Tracking RGB color can be defined as the problem of estimating the trajectory of an object in the image plane as it moves around a scene. The need for high power computers, the availability of high quality video cameras, usually we use ordinary webcams to handle videos. As the Kinect is getting cheaper now and people started using it in their applications, it is useful sometimes to use the RGB images generated from Kinect instead of the webcams, especially when your application is using depth information from Kinect. However, the increasing need for automated video analysis has generated a great deal of interest in object tracking algorithms. There are three key steps in video analysis, detection of interesting moving objects, tracking colors of such objects from frame to frame, and analysis of object tracks to recognize their behavior.

\subsection{Related Work}

A current paper on the topic discussed in this section has been written. However, we could find only few publications on detection and tracking of color in the images.

"Tajbakhsh and Grigat" also remarked the lack of literature on color chart detection. They proposed an algorithm for semiautomatic color chart detection in distorted images. The user initially selects the four chart corners in the image and the system estimates the position of all color regions using projective geometry. They transform the image with Sobel kernel, a morphological operator and thresholding into a binary image and find connected regions. They show the robustness of the method of distorted images

In 1999, at the University of British Columbia presented research titled "Tracking Color Objects in Real Time" and the goal of research is efficient tracking of color objects from a sequence of live images for use in real-time applications including surveillance, video conferencing and robot navigation.

"Dr.M.Sivabalakrishnan,K.Shanthi" They discussed in a paper title "person accounting system using EFV 
segmentation and fuzzy logic" This paper presents a novel method for people segmentation, they suggested a fuzzy-based approach for bringing together many inferred in image processing applications, The proposed method utilizes a fuzzy inference system. The algorithm uses background subtraction and based just on the edge flow-based foreground blocks to bring human segmentation. To processed object inference challenges in dynamic environments, they fused high-level defined and low-level advantages, and developed a fuzzy logic heuristic system for people tracking. Representative video sequences have been used to assess the result. The suggested people counting method are simply competent, and bring a real time functioning. They use a repetitive target detection scheme and motion prediction techniques that do not rely on spatial proximity.

\section{Method}

\subsection{RGB Image Representation in MATLAB}

In the RGB model, each color represents the basic color components Red, Green, and Blue. RGB color images are represented in the RGB color model as red, green and blue using 8-bit monochrome standard. The corresponding RGB color image has 24 bit/pixel -8 bit for each color band (red, green and blue). The RGB color represents to referring to arrow or column as a vector, it can be referred as a single pixel red, green and blue values as a color pixel vector $-(\mathrm{R}, \mathrm{G}, \mathrm{B})$. The color space representation is:
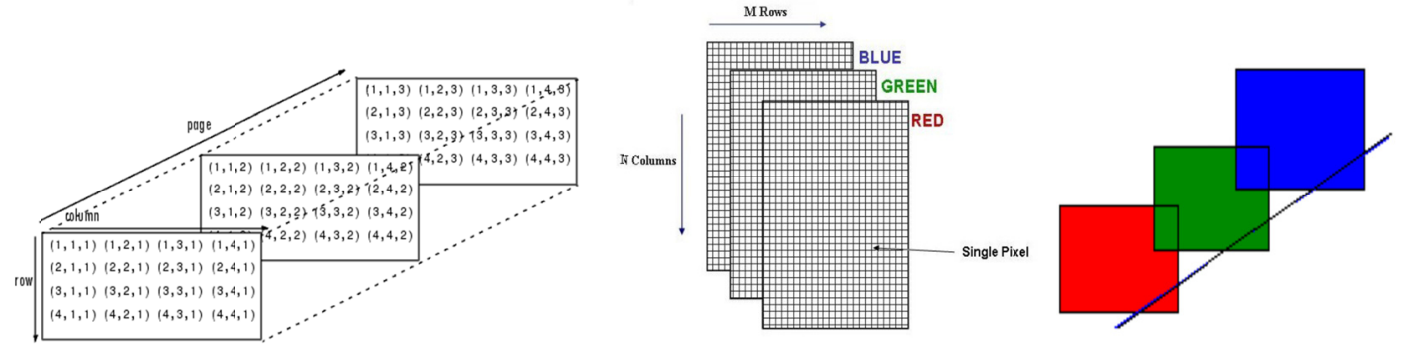

Figure 1. RGB Image Representation in MATLAB

\subsection{Kinect}

The physical parts of the Kinect sensor consist of cameras, a microphone array and an accelerometer. Additionally it provides a software pipeline that processes color, depth and skeleton data. The RGB camera can store colors with a resolution of 1280x960 pixels. The camera has an infrared (IR) emitter which emits infrared light beams and an IR depth sensor which makes it possible to read the emitted light and create a depth map from that information. The multi-array microphone contains four different microphones at different locations which provide audio recording capabilities which also can specify the location and specify the direction of the sound wave. A 3-axis accelerometer makes it possible to determine the current orientation of the Kinect device.

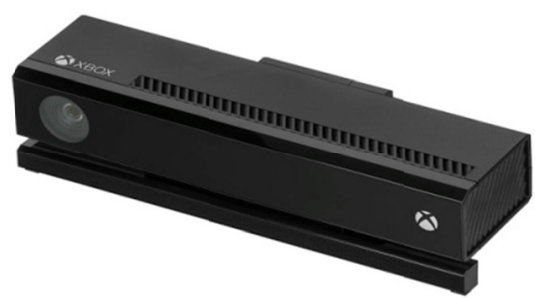

Figure 2. Kinect components

\subsection{Proposed Algorithm}

Several general purpose techniques and algorithms have been developed for RGB color tracking. In this paper color tracking and counting process primary colors of the input RGB frame, then each color is identified by its own pixel. The proposed algorithm discussed in this paper will be helpful in developing better and efficient algorithms in the field of tracking color. A basic flow chart diagram of the proposed algorithm is shown below 


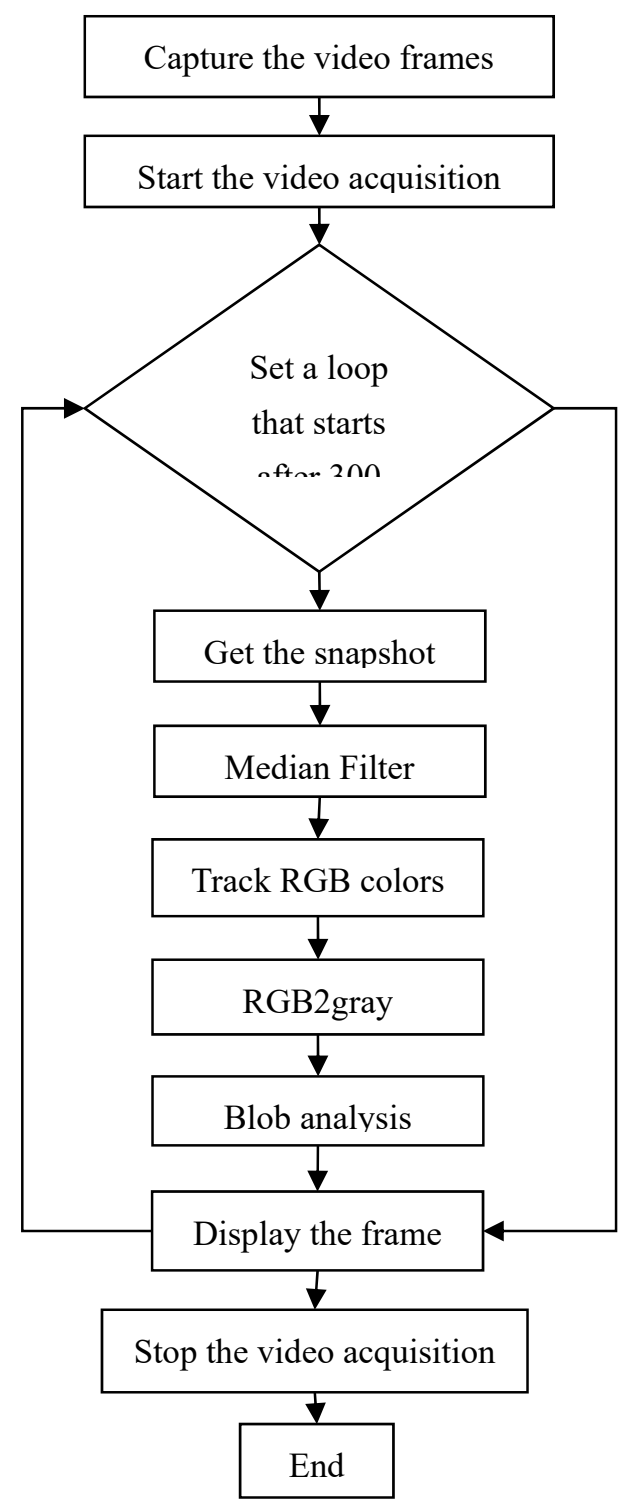

Figure 3. Detection and tracking techniques algorithms using color feature

The figure (3) present in the above flowchart are being explained the Kinect Capture the video frames using the video input function in MATLAB and Set the properties of the video object, Now to track color RGB objects in real time we have to subtract the color component from the grayscale image in the frame to extract the components in the image frame. And use a median filter to filter out noise and Convert the resulting grayscale image into a binary image MATLAB is built in "im2bw". Remove all those pixels less than what you want, Label all the connected components in the image. And we do the image blob analysis get a set of properties for each labeled region.

\subsubsection{Median Filter}

This class of filter belongs to the class of edge preserving smoothing filters which are non-linear filters. This means (1) that for two images $\mathrm{A}(\mathrm{x})$ and $\mathrm{B}(\mathrm{x})$ :

$$
\operatorname{median}[A(x)+B(x)] \neq \operatorname{median}[A(x)]+\operatorname{median}[B(x)]
$$

The median is just the middle value of all the values of the pixels in the neighborhood and median value becomes the new value for the central pixel. This must be taken into account if you plan on summing filtered images. Figure 5illustrates an example calculation. 


\begin{tabular}{|c|c|c|}
\hline 5 & 80 & 7 \\
\hline 18 & 3 & 10 \\
\hline 2 & 33 & 50 \\
\hline
\end{tabular}

\begin{tabular}{|c|c|c|}
\hline$*$ & $*$ & $*$ \\
\hline$*$ & 10 & $*$ \\
\hline$*$ & $*$ & $*$ \\
\hline
\end{tabular}

Neighborhood values: 2, 3,5,7,10,18,33,50,80

Median value: 10

Figure 5. Illustrates an example calculation

After detecting the RGB color in the input frame of the image, a median filter has been used to filter out the noise. A median filter is more effective than convolution when the goal is to simultaneously reduce noise.

The median filter performs well as long as the spatial density of the impulse noise is not large. However the adaptive median filtering can handle impulse noise with probabilities even larger than these.

\subsubsection{Blob Analysis}

Blob Analysis is a fundamental technique of machine vision based on analysis of consistent image regions, such as a tool of choice for applications in which the objects being inspected are clearly discernible from the background. Diverse set of Blob Analysis methods allows creating tailored solutions for a wide range of visual inspection problems.

\subsubsection{Motion detection}

Motion detection is the process of detecting a change in the position of an object relative to its surroundings or a change in the surroundings relative to an object. Motion detection can be achieved by either mechanical or electronic methods. When motion detection is accomplished by natural organisms, it is called motion perception. The MATLAB interface allows the user to define the commands to be performed at the run time. Once the user setup of the video source is complete the algorithm comes into play.

\subsubsection{Image segmentation}

In computer vision, image segmentation is the process of dividing a digital image into multiple segments collection of pixels, also known as super pixels. The target of segmentation is meaningful and changes the representation of an image into anything that is more meaningful and easier to analyze. Image segmentation is generally used to locate objects and boundaries lines, curves... etc in images. More precisely, image segmentation is the process of assigning a label to all pixels in an image such that pixels with the same label share specific attributes.

\subsection{Color-Depth Segmentation}

We explain the results using only the color segmentation algorithm. The test consists in several kinds of object RGB colors as is shown in Figure $8(\mathrm{a}, \mathrm{d}, \mathrm{g})$ detection and tracking red ,green and blue color feature The corresponding color segmented image when we select the red, blue and green colors is depicted in. Figure $8(\mathrm{~b}, \mathrm{e}, \mathrm{h})$ segmentation red, green and blue colors. By examining this figure, we note that the color of the selected object is highlighted in black, being the rest of the objects represented in gray scale. The objects colors image shown in the Figure 8 (c,f,i) detection and tracking red, green and blue color in depth Kinect is transformed in the RGB color space as described in the previous section. This transformation simplifies the color segmentation and it is only a matter of computing the Euclidean distance for each component to the point of interest. The proposed System architecture for image segmentation using background subtraction and incremental version of detection and tracking motion, color-Depth Segmentation technique shown below in Figure 6. and the result is shown in figure $8(\mathrm{c}, \mathrm{f}, \mathrm{i})$ detection and tracking red color in depth Kinect. 


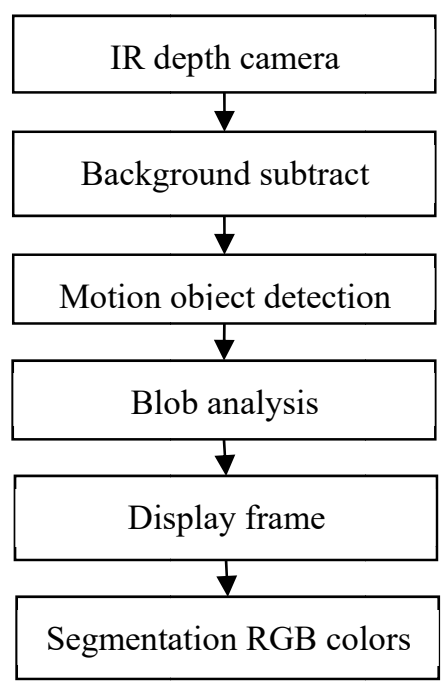

Figure 6. Detection and tracking motion Color-Depth Segmentation

\subsubsection{Background Subtraction}

Moving object detection and tracking is an important and fundamental topic in computer vision. Its applications can be found in a large number of engineering fields, including One of the most commonly used methods to detect moving objects is background subtraction. Classic background subtraction approaches work by modeling the scene based on the pixel distributions. There has been a lot of research done in this field to model backgrounds ranging from simple static ones to complex dynamic cases. Probably the most widely used method in this area are the Mixture output of Gaussians (MoG) approach by "Stauffer and Grimson". It describes the values of each background pixel throughout the sequence with a mixture of Gaussian distributions.

In background subtraction absolute difference is taken between every current image I $t(x, y)$ and the reference background image $\mathrm{B}(\mathrm{x}, \mathrm{y})$ to find out the motion detection mask $\mathrm{D}(\mathrm{x}, \mathrm{y})$. The (2) reference background image is generally the first frame of a video, without containing foreground object.

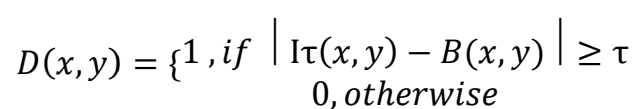

$\mathrm{D}(\mathrm{x}, \mathrm{y})=$ motion detection

I $t(x, y)=$ current image

$\mathrm{B}(\mathrm{x}, \mathrm{y})=$ background image

$\mathrm{T}=$ threshold

Where a threshold, which decides whether the pixel is foreground or background. If the absolute difference is greater than or equal to $\tau$, the pixel is classified as foreground; otherwise the pixel is classified as background.

\section{Results}

We have worked on real-time tracking red, green and blue colors, to track these three colors in the video. The proposed algorithm explained above applies to the video frames to achieve the required tracking RGB color. The results obtained are shown below:

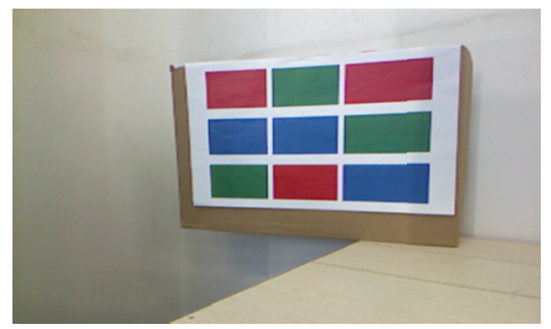

Figure 7. Test chart colors 


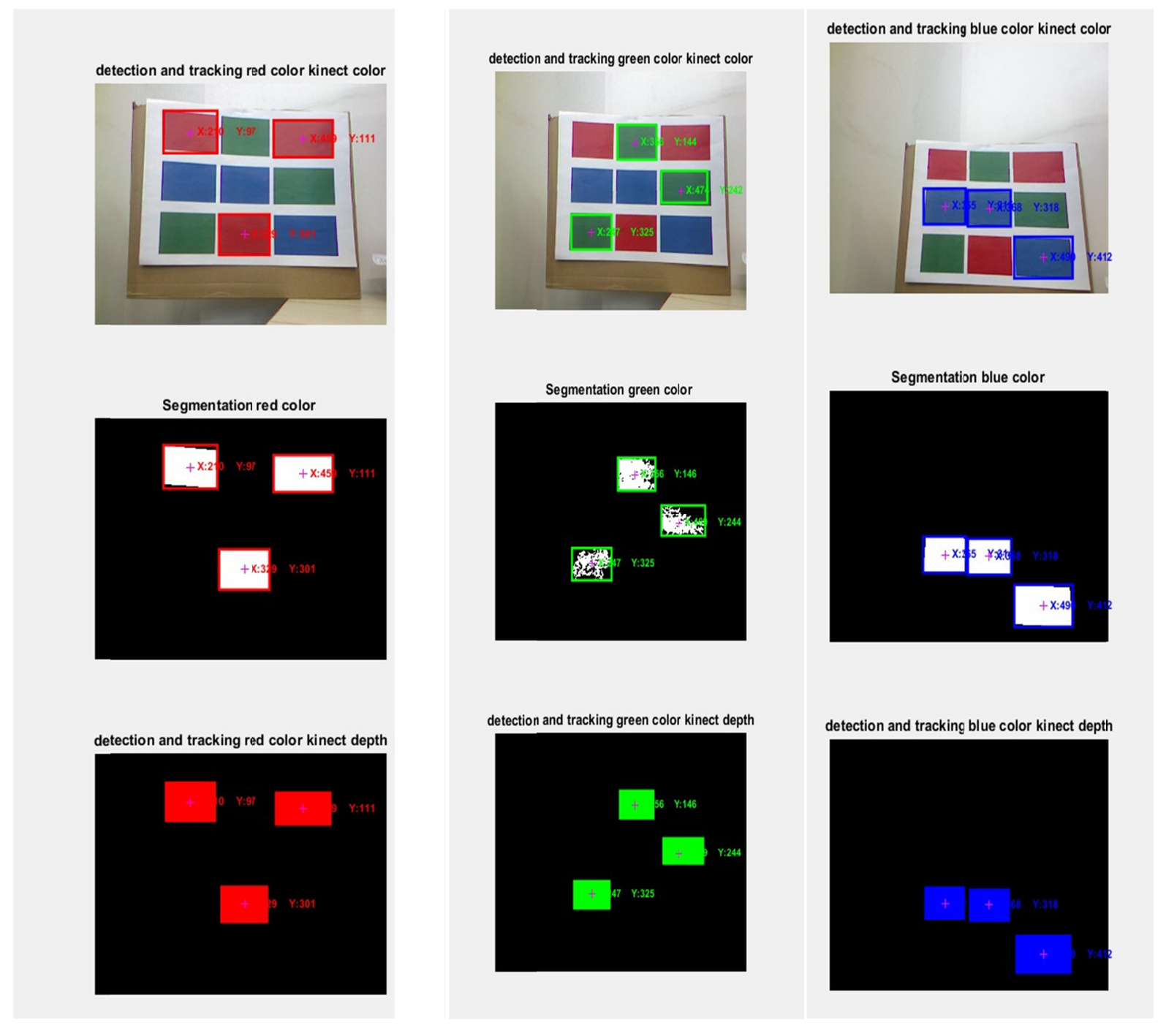

from up to down $(\mathrm{a}, \mathrm{b}, \mathrm{c})(\mathrm{d}, \mathrm{e}, \mathrm{f})(\mathrm{g}, \mathrm{h}, \mathrm{i})$

Figure 8. (a)detection and tracking red color feature.(b)segmentation red color.(c) detection and tracking red color in depth Kinect (d)detection and tracking green color feature.(e)segmentation green color.(f) detection and tracking green color in depth Kinect (g)detection and tracking blue color feature.(h)segmentation blue color.(i) detection and tracking blue color in depth Kinect

\section{Conclusion}

Commonly, In this paper, the algorithm has been developed for real time detection and tracking RGB color based on kinect .Tracking of the RGB colors is done on the basis of region properties such as centered, bounding box etc. Most of the time median filtering is used in image processing to remove noise during real-time tracking RGB color. We used Median filtering because it is far better the convolution technique when the aim to prevent edges and to eliminate noise, anything relatively small in size compared to the size of the neighborhood will have minimal affect on the value of the median, and will be filtered out also the median filter is more costly to count than a smoothing filter or other filters. In video processing applications, variants of the background subtraction method are broadly used for the detection of moving objects in video sequences. The background subtraction is the most popular and common approach for motion detection.

\section{Acknowledgments}

Fund projects: major national science and technology support program project (2012BAF 12B22), Jilin provincial key scientific and technological project (20140204050GX), Jilin province, a major scientific and 
technological projects (2012ZDGG004).

\section{References}

Andreas Ernst, A. P., (2008). Tobias Ruf and Jens-Uwe Garbas, Check My Chart: A Robust Color Chart Tracker for Colorimetric Camera Calibration. Electronic Imaging Department Am Wolfsmantel, Erlangen, Germany, 33.

Barghout, L., Lawrence, \& Lee, W. (2003). Perceptual information processing system. Patent Application.

Gao., Q. W. A. .Z. (2008). Study on a Real-Time Object Tracking System. International Symposium on Computer Science and Computational Technology ISCSCT.

Grigat, T. T. A. R. R. (2008). Automatic detection of colour charts in images. Pattern Recognition and Applications, Innsbruck, Austria, 347-352.

Hillman, J., (2013). Kinect in retro games School of Computing Blekinge Institute of Technology, Karlskrona, Sweden.

http://docs.adaptive-vision.com/4.2/studio/machine_vision_guide/BlobAnalysis.html, BlobAnalysis. 2015.

http://homepages.inf.ed.ac.uk/rbf/HIPR2/median.html, Median filter. 2015.

http://www.mathworks.com/discovery/image-segmentation.html, image segmentation. 2015.

http://www.webopedia.com/TERM/M/motion_detection.html, motion_detection. 2015.

https://dev.windows.com/en-us/kinect, kinect. 2015.

https://en.wikipedia.org/wiki/Image_segmentation, Image segmentation. 2015.

https://en.wikipedia.org/wiki/Motion_detection, Motion detection. 2015.

Huang, S. C. (2010). A new scheme for generating initial palettes of color quantized images. International Journal of Innovative Computing, Information and Control, 6(7), 3011-3022.

Kravtchenko, V. (1999). Tracking Color Objects in Real Time. 1999.

Lee, Y. S. L. M. Z. R. S. J. (2010). Applying Self-Constructing Clustering to Color Image Quantization. National Sun Yat-sen University Institutional Repository ICIC Express Letters, 3(3), (B)(No. 3(B)): p. 818.

Liu, C. C. (2012). A global color transfer scheme between images based on multiple regression analysis. International Journal of Innovative Computing, Information and Control (IJICIC), 8(1), (ISSN 1881-803 X), 167-186.

M.Manikandan, R. B. (2015). Real time People Tracking and counting system using Fuzzy Logic. IJESC:International Journal of Engineering Science and Computing, 4(2321-3361), 1222-1229.

Makwana, M. B. A. (2015). DETECTION Background Subtraction Technique As Review. International Journal of Advance Engineer ing and Research Development (IJAERD), 4, 10-13.

Piccardi, M. (2004). Background subtraction techniques: A review. IEEE International Conference on Systems, Man and Cybernetics.

Shing, C. W. Y. A. L. T. (2011). 3D dance head using kinect and $3 d$ projector. Department of computer and science and engineer, China University of Hong Kong.

Stauffer, C., Grimson, W. E. L. (1999). Adaptive background mixture models for real-time tracking. IEEE Computer Society Conference, 2, 246-252.

Stockman, L. G. S. A. G. C. (2015). Computer Vision. International Journal of Engineering Research and Applications, 5(5), 279-325.

\section{Copyrights}

Copyright for this article is retained by the author(s), with first publication rights granted to the journal.

This is an open-access article distributed under the terms and conditions of the Creative Commons Attribution license (http://creativecommons.org/licenses/by/4.0/). 